\title{
Kernos
}

Revue internationale et pluridisciplinaire de religion grecque antique

$15 \mid 2002$

Varia

\section{Daniela CASTALDO, Il Pantheon Musicale. Iconografia nella ceramica attica tra VI e IV secolo}

\section{Alexandra Goulaki-Voutira}

\section{OpenEdition \\ Journals}

Édition électronique

URL : http://journals.openedition.org/kernos/1435

DOI : 10.4000/kernos. 1435

ISSN : 2034-7871

\section{Éditeur}

Centre international d'étude de la religion grecque antique

Édition imprimée

Date de publication : 1 janvier 2002

ISSN : 0776-3824

\section{Référence électronique}

Alexandra Goulaki-Voutira, « Daniela CASTALDo, II Pantheon Musicale. Iconografia nella ceramica attica tra VI e IV secolo », Kernos [En ligne], 15 | 2002, mis en ligne le 16 juin 2011, consulté le 24 septembre 2020. URL : http://journals.openedition.org/kernos/1435; DOI : https://doi.org/10.4000/kernos. 1435 
style enlevé, la documentation très riche et les textes abondamment traduits (mais trop souvent sans références...). Il ne s'agit pas d'un $x^{\text {e }}$ livre sur les femmes grecques, venant après tant d'autres, compenser enfin le silence, long et obstiné, d'une certaine historiographie. Il s'agit d'un très beau livre, généreux, haut en couleurs, qui donne véritablement vie aux femmes grecques, à défaut d'avoir vraiment la possibilité de leur donner la parole puisque les intermédiaires de notre information sont presque toujours masculins. Il s'agit aussi d'un exercice de méthode historique, même si l'originalité du ton rompt délibérément avec le style habituel de la synthèse en histoire.

C'est du côté du religieux que s'ouvre le propos avec «Le féminin, les femmes et le sacré ». D'une interrogation sur le divin féminin en passant par les prêtrises et les rituels spécifiques (Déméter, Dionysos), il ressort que « la religion constitue un révélateur à la fois précis et puissant du féminin grec ». Puis l'on en vient aux portraits épiques, de Briséis et Chryséis à Pénélope en passant par Nausicaa, mais sans Hélène. L'étude des mots pour dire la femme, la fille, la fille de... est particulièrement éclairante. Le chapitre suivant traite « Du corps et du comportement ", passant ainsi de l'épopée à la biologie d'Aristote et des méde cins : polarité sexuelle, sexualité et fécondation sont autant de lieux où s'exprime une lecture masculine, idéologiquement marquée, du corps des femmes. Après le biologique, le social: un parcours dans l'oikos de Périclès ouvre à la compréhension des pratiques d'échange matrimonial entre grandes familles et à celle des questions d'âge au mariage, d'amours sexuellement différents, de filles et de garçons et, en intermezzo, de rites nuptiaux. Puis c'est l'expérience de l'Ischomaque de Xénophon face à sa toute jeune épouse qui est finement décrite, pour en arriver aux Aspasie et autres Nééra, les courtisanes de haut vol, hors du système de 1' « endogamie politique », La toute dernière partie s'intitule «Comment les quitter?": sur un poème de Sappho, bien sûr ! Une très brève bibliographie presque exclusivement francophone vient en fin d'ouvrage et s'achève par la mention de trois thèses patronnées par l'A. et non encore publiées. Un beau geste d'enseignant.

P. Brulé nous montre avec empathie qu'après une sorte de nécessité pour les femmes grecques de trouver des interprètes féminines dans le monde des historiens contemporains (les gender studies furent d'abord féministes, puis féminines), un porte-parole masculin leur rend aujourd'hui pleinement et joliment justice.

Vinciane Pirenne-Delforge (Université de Liège)

\section{Daniela CASTALdo, Il Pantheon Musicale. Iconografia nella ceramica attica} tra VI e IV secolo, Ravenna, Longo Editore, 2000. 1 vol. $17 \times 24 \mathrm{~cm}, 325$ p., 174 fig. ISBN : 88-8063-213-2.

Ce livre est la publication d'une thèse de doctorat, rédigée à l'Université de Lausanne sous la direction du professeur Claude Bérard. L'A. part du constat que les témoignages iconographiques sur la musique grecque antique offrent une image différente de celle que nous donnent les sources écrites. Cette divergence est encore plus nette, à son avis, dans la musique des dieux. Aussi rassemble-t-elle et présente-t-elle dans son livre les représentations de la musique du panthéon olympien dans la céramique attique du $\mathrm{vI}^{\mathrm{e}}$ au $\mathrm{Iv}^{\mathrm{e}}$ siècle av. J.-C. Elle organise son sujet autour des figures d'Apollon et de Dionysos en distinguant deux genres de musique, dont elle définit le caractère différent : la musique apollinienne, d'une part, et la musique associée à Dionysos et son entourage, de l'autre. Sa discussion comprend aussi d'autres divinités, dont les liens avec la musique sont moins 
évidents ou moins directs, comme Artémis, Athéna, Hermès et Éros. À travers cette optique, elle précise la signification des instruments musicaux les plus importants et les plus répandus dans la Grèce ancienne en tenant compte, plus que de leurs caractéristiques organologiques, du contexte dans lequel ils apparaissent, des personnages qui les jouent ainsi que du caractère de leur musique. Elle emploie la méthode de l'analyse iconographique de ses maîtres qu'elle définit comme celle de "l'école de Lausanne » et de "l'école de Paris » (p. 6). Cette méthode conduit à une approche différente de celle des historiens musicologues, qui se fondent surtout sur les témoignages écrits. L'A. utilise un grand nombre de représentations puisées dans les volumes du CVA, les catalogues d'expositions et de collections publiques et privées ainsi que dans les actes de congrès et diverses études spécialisées. Elle a mené des recherches fructueuses dans les archives iconographiques de l'Institut d'Archéologie et d'Histoire Ancienne de l'Université de Lausanne et dans les archives Beazley à Oxford.

Le livre est articulé en deux parties. Dans la première sont examinées les scènes musicales relatives à Apollon et à Dionysos, conçus comme deux pôles opposés; entre les deux se trouvent insérées les représentations de musique relatives à Artémis, à Athéna, à Hermès et à Éros. Apollon, le seul vrai musicien parmi les dieux de l'Olympe, apparâit régulièrement dans certaines scènes, comme l'assemblée des dieux, les noces de Pélée et de Thétis, la naissance d'Athéna, l'apothéose d'Héraclès, appartenant au répertoire de la poésie épique et des rhapsodes, qui en sont la source et le modèle.

L'A. analyse le caractère de la musique apollinienne et la relation de la lyre avec l'arc. Elle met en relation l'image d'Apollon poète et chanteur avec celle d'Héraclès citharède; elle se tourne ensuite vers les scènes de libation, les processions de dieux, la relation d'Apollon avec les doubles auloi, pour terminer avec le concours d'Apollon et de Marsyas et sa valeur symbolique. Ensuite, elle examine la relation d'Artémis, d'Athéna et d'Hermès avec la musique. Ceci lui donne l'occasion de s'occuper du rapport particulier de ses divinités avec certains instruments, comme la trompette, les crotales, la cithare, ainsi que de leur rôle dans divers contextes. Elle continue avec l'examen des scènes musicales relatives à Éros, des scènes d'école, de l'éducation des jeunes, d'Éros parmi les femmes (poésie érotique) et enfin d'Éros en compagnie de Dionysos et d'Aphrodite. La dernière section de la première partie du livre est dédiée à Dionysos. La plupart du temps, Dionysos ne joue pas lui-même de la musique, mais il inspire le cortège de satyres et de ménades qui le suit. L'instrument principal que jouent ceux-ci sont les doubles auloi. L'A. ordonne de manière systématique un grand nombre de représentations dionysiaques en distinguant des ensembles: cortège de Dionysos avec instruments musicaux, scènes dionysiaques sans la présence du dieu, le tambour, les cymbales et les instruments à cordes dans la céramique du $\mathrm{IV}^{\mathrm{e}}$ siècle av. J.-C.

Dans la deuxième partie du livre, l'A. suit la pénétration dans le domaine de Dionysos de sujets provenant du cycle iconographique d'Apollon et vice versa. On rencontre ainsi des satyres citharèdes accompagnant Dionysos, en même temps qu'Apollon est présent au banquet de Dionysos et d'Ariane ou au retour d'Héphaistos sur l'Olympe. On voit aussi, à côté d'Apollon, des figures féminines debout et immobiles, normalement par paires, faisant résonner des cymbales. L'identité de ces figures est à chercher parmi les Muses, les Nymphes ou d'autres divinités de la nature. Ensuite sont examinés les rapports entre Apollon et Dionysos dans les sources littéraires, les plus anciennes apparitions communes des deux divinités dans l'iconographie, leur présence complémentaire sur les deux faces d'un vase, leur rapprochement dans les représentations de la fin du ve et du 
$\mathrm{rv}^{\mathrm{e}}$ siècle. Elle observe que nous assistons souvent, pendant cette période, à une "dionizzazione » ou à une " apollinizzazione » de divers sujets et termine son tour d'horizon avec Dionysos musicien. Ce ne sont pas les instruments musicaux qui déterminent le caractère d'une scène, mais le genre et le style de la musique qu'on y joue, qu'on ne peut reconnaître qu'à travers le contexte iconographique.

Cette recherche porte la marque de savants maîtres, bien visible dans la connaissance étendue et approfondie du matériel et dans l'attention prêtée au contexte dans le processus d'interprétation. On découvre souvent avec plaisir de belles observations de détail concernant des scènes musicales peu connues, autant de petites trouvailles qui ne sont pas reflétées dans les conclusions. C'est là une certaine injustice à l'égard de son sujet. Une vue d'ensemble aussi large comporte forcément peu d'éléments spectaculaires et semble manquer d'originalité. Mais en examinant les chapitres de plus près, on découvre une richesse d'observations, de remarques et de choix qui surprennent agréablement et ampli fient nos connaissances au sujet de la musique antique par l'examen des détails. Très utiles sont le classement des scènes dionysiaques, les observations sur les instruments à percussion, sur la syrinx, ainsi que sur certaines particularités iconographiques. Sur certains points, on peut signaler des lacunes bibliographiques, surtout en ce qui concerne le domaine de l'iconographie musicale dont une meilleure connaissance aurait sans doute contribué à rendre cette étude plus complète. Dans la discussion de sujets tels que le concours de Marsyas et d'Apollon (p. 35), celui de Thamyris et des Muses (p. 21), Orphée ou les femmes musiciennes (p. 72-73), l'A. semble ignorer une série d'articles publiés dans la revue spéciale d'iconographie musicale Imago Musicae, en particulier dans les volumes VII (1990) et VIII (1991) ou encore les actes du congrès de Hambourg, H. Heckmann et al. (éd.), Musikalische Ikonographie (Laaber Verlag, 1994). Dans la présentation du sujet, elle n'a pas pu éviter un certain nombre de redites (p. ex. le concours d'Apollon et de Marsyas, p. 35 et 55, 58). Sa lecture des images présente parfois des erreurs (elle ne reconnaît pas l'accordage, p. 41 et fig. 18) ou des interprétations trop littérales de certaines scènes (p. 22, la musique cadencée dans les processions des dieux), bien qu'elle reconnaisse pour la plupart correctement les conventions iconographiques. Un autre point difficile est son traitement un peu superficiel des Muses, des Nymphes et des Ménades comme divinités de la nature (p. 123-137). Toutefois ces remarques n'ont qu'une importance secondaire et ne diminuent pas l'intérêt de cette étude, dont les propositions ouvertes au dialogue et les découvertes conduisent à une meilleure compréhension du rôle de la musique dans la Grèce ancienne.

Ce livre présente un grand intérêt pour le musicologue, l'archéologue, l'historien, l'organologue et enfin pour tous ceux qui s'intéressent généralement à l'Antiquité classique.

Alexandra Goulaki Voutira (Département de Musique Université Aristote de Thessalonique)

Annette Hupfoher, Kulte im kaiserzeitlichen Sparta. Eine Rekonstruktion anband der Priesterämter, Berlin, Akademie Verlag, 2000. 1 vol. 17,5×24,5 $\mathrm{cm}, 245$ p. ISBN : 3-05-003548-X

Jusqu'il y a peu, l'histoire de Sparte à l'époque romaine n'avait guère suscité l'intérêt des chercheurs et était au mieux traitée comme l'ultime étape du déclin spartiate. Ainsi que le souligne l'A. en introduction, cette tendance s'observait également dans le domaine de l'histoire religieuse. En effet, dans leurs travaux fondamentaux, S. Wide (Lakonische Kulte, Leipzig, 1893) et L. Ziehen (art. "Sparta 